Please do not remove this page

RMIT

UNIVERSITY

\title{
Conductometric hydrogen gas sensor based on polypyrrole nanofibers
}

Al-Mashat, Laith; Tran, Henry; Wlodarski, Wojciech; Kaner, Richard; Kalantar Zadeh, Kourosh https://researchrepository.rmit.edu.au/esploro/outputs/9921863523501341/filesAndLinks?institution=61 RMIT_INST\&index=null

Al-Mashat, L., Tran, H., Wlodarski, W., Kaner, R., \& Kalantar Zadeh, K. (2008). Conductometric hydrogen gas sensor based on polypyrrole nanofibers. IEEE Sensors Journal, 8(4), 365-370.

https://doi.org/10.1109/jsen.2008.917476

Published Version: https://doi.org/10.1109/jsen.2008.917476

Repository homepage: https://researchrepository.rmit.edu.au

(c) 2008 IEEE. Personal use of this material is permitted. However, permission to reprint/republish this material for advertising or promotional purposes or for creating new collective works for resale or redistribution to servers or lists, or to reuse any copyrighted component of this work in other works must be obtained from the IEEE.

Downloaded On 2023/04/26 23:56:59 +1000 


\title{
Conductometric Hydrogen Gas Sensor Based on Polypyrrole Nanofibers
}

\author{
Laith Al-Mashat, Member, IEEE, Henry D. Tran, Wojtek Wlodarski, Member, IEEE, Richard B. Kaner, and \\ Kourosh Kalantar-Zadeh, Member, IEEE
}

\begin{abstract}
Polypyrrole nanofibers are synthesized through a template-free chemical route and used as the active component for hydrogen gas sensing at room temperature. The synthesis of polypyrrole nanofibers was achieved by using bipyrrole as an initiator to speed up the polymerization of pyrrole with $\mathrm{FeCl}_{3}$ as the oxidizing agent. Scanning and transmission electron microscopy studies indicate that the resulting polypyrrole forms a nanofibrous mat with average nanofiber diameter of $18 \mathrm{~nm}$. Fourier transform infrared spectroscopy and elemental analysis confirms that the structure of the nanofibers is comparable to bulk polypyrrole. Gas sensing properties of polypyrrole nanofibers were investigated by depositing nanofiber dispersions on an interdigited conductometric transducer. The sensor performance was tested through programmable exposure towards different concentrations of hydrogen gas diluted in synthetic air in an environmental cell at different temperatures. A short response time of $43 \mathrm{~s}$ was observed upon exposure to a concentration of $1 \%$ hydrogen with a decrease in film resistance of $312 \Omega$ at room temperature. The sensor sensitivity was analyzed with gradual elevation of the operating temperature.
\end{abstract}

Index Terms-Conducting polymers, hydrogen sensors, polypyrrole nanofibers.

\section{INTRODUCTION}

C ONDUCTING polymers such as polypyrrole, polyaniline, polythiophene, and their derivatives have been investigated for gas sensing applications for more than 20 years [1]. Gas sensors based on conducting polymers have many features that are superior to their metal oxides counterparts including high sensitivities, short response time, and room temperature operation. In contrast, metal oxides generally operate at elevated temperatures around $300{ }^{\circ} \mathrm{C}$ [2]. Conducting polymers are easily synthesized through chemical or electrochemical

Manuscript received October 31, 2007; revised January 4, 2008; accepted January 8, 2008. This work was supported in part by the Microelectronics Advanced Research Corporation (MARCO) and its Focus Center Research Program on Functional Engineered NanoArchitectonics (FENA) and in part by the U.S. National Science Foundation under Grant DMR-0507294 (R.B.K.). The associate editor coordinating the review of this paper and approving it for publication was Prof. Evgeny Katz.

L. Al-Mashat, W. Wlodarski, and K. Kalantar-Zadeh are with the Sensor Technology Laboratory, School of Electrical and Computer Engineering, RMIT University, GPO Box 2476V, Melbourne 3001, Australia (e-mail: laith.almashat@ieee.org;ww@rmit.edu.au; kourosh.kalantar@rmit.edu.au).

H. D. Tran and R. B. Kaner are with the Department of Chemistry and Biochemistry and California NanoSystems Institute, University of California, Los Angeles, CA 90095-1569, USA (e-mail: htran@chem.ucla.edu; kaner@chem. ucla.edu).

Color versions of one or more of the figures in this paper are available online at http://ieeexplore.ieee.org.

Digital Object Identifier 10.1109/JSEN.2008.917476 processes, and new polymers can be designed by the substitution of different functional groups onto the polymer backbone.

Polypyrrole and its derivatives (PPy) are formed through the oxidation of pyrrole or substituted pyrrole monomers. The oxidation is usually carried out by either electropolymerization on a conductive substrate (electrode) with the application of an external potential, or by chemical polymerization in solution through the use of a chemical oxidant. The two methods produce PPy with different chemical and electrical properties [3]. Changes to the electrical conductivity of PPy have been noted upon exposure to various organic and inorganic gases. Blanc et al. [4] measured the conductivity of PPy films obtained by electropolymerization method under exposure to $\mathrm{O}_{2}, \mathrm{NH}_{3}$, $\mathrm{NO}_{2}$ diluted in synthetic air or $\mathrm{N}_{2}$ at $100{ }^{\circ} \mathrm{C}$. It was established that a wide range of organic vapors can alter the conductivity of PPy, which facilitated its application in field effect transistor (FET)-based gas sensors [5]-[8]. A CO sensor was fabricated by Liu et al. [9] by growing a PPy film on the surface of an interdigitated-capacitive transducer using the electropolymerization technique. Recently, Waghuley and coworkers [10] reported a $\mathrm{CO}_{2}$ sensor based on PPy film synthesized using chemical polymerization of the pyrrole monomer.

Although PPy thin films can be very easily made by electrochemical deposition, their performance as gas sensing films is affected by the fact that the active sensing components remain embedded in the bulk, which limits both the efficiency and the sensitivity. This can be improved by making PPy in a nanofiber form to generate high surface area for a given mass or volume, which can enhance the sensitivity by providing a porous structure that has better interactions between the sensing film and the target gas molecules [11]. In order to enhance the chemical sensing properties of PPy, an extensive amount of research work has concentrated on the synthesis of nanostructured forms of PPy. The production of nanostructured PPy has been studied using templated synthetic methods employing mesoporous silica, anodized aluminum oxide membranes, and particle track-etched membranes [12], [13]. A bulk growth approach using $\mathrm{V}_{2} \mathrm{O}_{5}$ seeds as a template has also been reported recently [14]. The method of surfactant-mediated synthesis, which has been so successful in the synthesis of polyaniline nanofibers yields only nonfibrous, granular powders in the case of polypyrrole [15]. Fibrillar and tubular morphologies of polypyrrole has been observed when $\beta$-naphthalene sulfonic acid (NSA) or p-toluenesulfonate acid (TsOH) is used as a dopant during the synthesis process [16]. These fibers and tubes have diameters in the range of 50-2000 nm and are formed presumably as a result of the solution aggregation of the dopant anions. In general, 


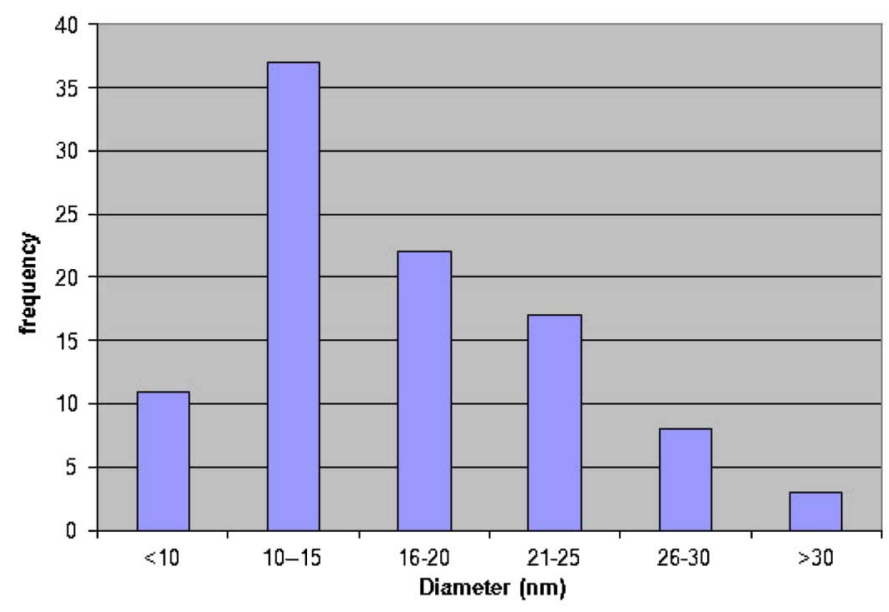

Fig. 1. Distribution of PPy nanofiber diameters resulting from a template-free synthesis using bipyrrole as an initiator.

a straightforward bulk synthesis of nanofibers of polypyrrole directly from pyrrole monomer with average fiber diameter less than $100 \mathrm{~nm}$, has been difficult to achieve.

The application of nanostructured conducting polymers in gas sensing has so far focused on polyaniline nanofibers [17], [18]. Polyaniline nanofiber films outperform their convention counterparts in terms of sensitivity and response time due to their higher surface to volume ratios, which provide better interaction between the film and the target gas molecules. According to the best of the authors' knowledge, this paper is the first study on PPy nanofibers thin films investigated for hydrogen gas sensing applications.

Here, we report the fabrication of a hydrogen gas sensor from polypyrrole nanofibers deposited on to conductometric transducers. Conductivity changes of the sensitive films upon exposure to different concentrations of hydrogen gas under variable operating temperature is measured.

\section{SYNTHESIS AND CHARACTERIZATION OF POLYPYRROLE NANOFIBERS}

\section{A. Synthesis}

All chemicals were purchased from Sigma-Aldrich. Monomers were distilled prior to use. Bipyrrole was synthesized according to established procedures [19]. Reactions were performed in $20 \mathrm{ml}$ glass vials in which pyrrole $(50 \mathrm{mg}$, $0.74 \mathrm{mmole}$ ) and bipyrrole (3 $\mathrm{mg}, 0.023 \mathrm{mmol}$ ) were dissolved in $10 \mathrm{~mL}$ of methanol and rapidly mixed with a separate solution of $\mathrm{FeCl}_{3}(120 \mathrm{mg}, 0.74 \mathrm{mmol})$ in $10 \mathrm{ml}$ of deionized water. The reaction mixture was vigorously shaken for several seconds and then left unagitated for one day. The crude product was purified by centrifugation and washed multiple times with deionized water. The purified product is typically resuspended in deionized water to a concentration of $2 \mathrm{~g} / \mathrm{l}$. The resulting nanofibrillar morphology of PPy from this synthetic process was found to have a statistical distribution of nanofibrillar diameters, as shown in Fig. 1. The mechanism of polypyrrole nanofiber formation is described in great detail elsewhere [20].

\section{B. Scanning Electron Microscope (SEM) and Transmission Electron Microscopy (TEM) Analysis}

The morphology of PPy nanofibers was analyzed with a JEOL JSM-6700F Field Emission Scanning Electron microscope by drop casting a $\sim 2 \mathrm{~g} / \mathrm{l}$ dispersion of PPy nanofibers onto a silicon wafer and allowing it to dry in a clean room environment.

Transmission electron microscopy (TEM) (JEOL 100CX) was also used to characterize the PPy nanofibers. A scanning electron microscope (SEM) and a TEM [Fig. 2(a) and (b)] show that a nanofibrous mat of PPy has been created. The PPy nanofibers have an average diameter of $18 \mathrm{~nm}$ based on statistical analysis of the TEMs (Fig. 1) with lengths on the order of several micrometers.

\section{Elemental Analysis}

Elemental analysis (conducted with a Thermo Electron/ FlashEA 1112 Elemental Analyzer) reveals that the ratio of $\mathrm{C} / \mathrm{N}$ of the polypyrrole nanofibers (3.5) is consistent with the theoretical $\mathrm{C} / \mathrm{N}$ ratio of pure polypyrrole (3.43) and lends further support that the observed nanofibers are indeed polypyrrole.

\section{Fourier Transform Infrared Spectroscopy (FTIR) Analysis}

Fourier transform infrared spectroscopy (FTIR) was used to analyze dispersions of PPy nanofibers. FTIR spectra were obtained with a JASCO FT/IR-420 using pressed potassium bromide $(\mathrm{KBr})$ pellets. The spectrum shown in Fig. 3 reveals the characteristic bands of PPy nanofibers. Absorption bands are observed at 1488 and $1558 \mathrm{~cm}^{-1}$ (stretching vibrations of the pyrrole ring), 917 and $1200 \mathrm{~cm}^{-1}$ (stretching vibrations of doped PPy), and 1045 and $1315 \mathrm{~cm}^{-1}$ (C-N stretching vibrations and $\mathrm{C}-\mathrm{H}$ deformations, respectively) indicating that the PPy nanofibers share the same molecular structure as that of established methods for making PPy [21].

\section{EXPERIMENTAL}

\section{A. Sensor Fabrication}

The sensor consists of two physical layers, namely, a sensitive thin film of PPy nanofibers that interact with the target gas through redox reactions and a conductometric transducer that transforms this reaction into an electrical signal to be captured by a computerized monitoring system.

For the transducer fabrication, platinum was sputtered to form the interdigitated electrode system on the front side and the heater resistor on the backside. Polished sapphire $\left(\mathrm{Al}_{2} \mathrm{O}_{3}\right)$ was used as a substrate because of its excellent electrical insulating and heat conducting properties. An intermediate $30 \mathrm{~nm}$ thick layer of titanium was used to improve the adhesion of the platinum layer to the sapphire substrate. The structure was fabricated using a standard liftoff technique. A schematic diagram of the conductometric transducer utilized is shown in Fig. 4.

The sensing layer was formed by drop-casting a PPy nanofiber dispersion onto the interdigital fingers of the transducer surface. Afterwards, the solution was allowed to evaporate in a clean environment for one day. The thicknesses of the resulting PPy nanofiber thin films were measured and found to be $\sim 0.3 \mu \mathrm{m}$. 

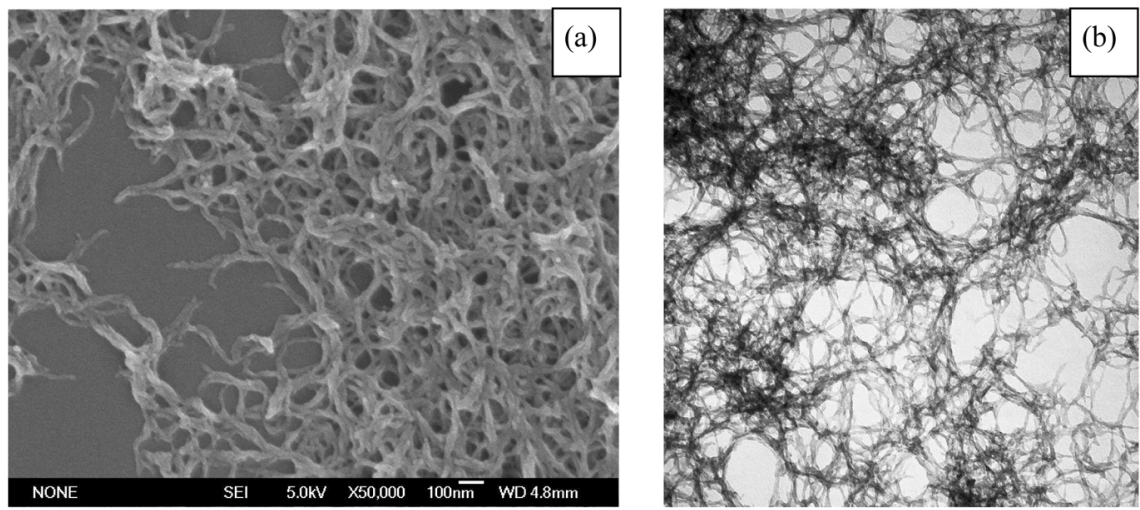

Fig. 2. (a) Scanning and (b) transmission electron micrographs of PPy nanofibers.

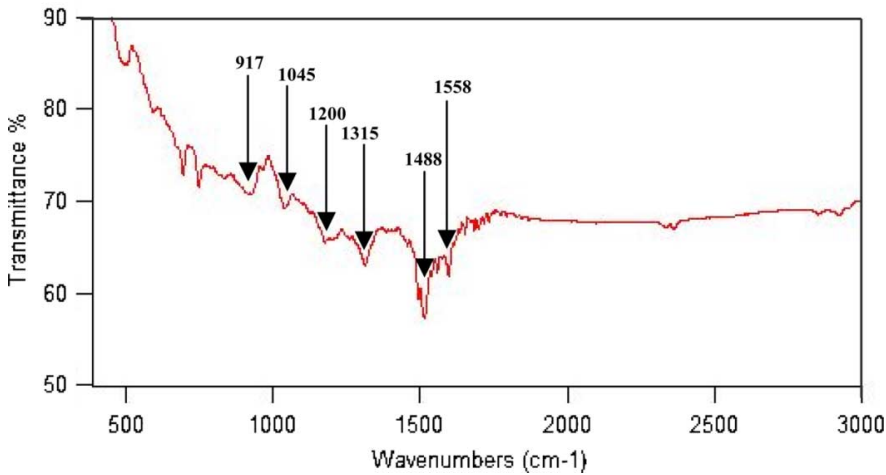

Fig. 3. FTIR spectrum of PPy nanofibers obtained by the template-free synthesis using bipyrrole as an initiator.

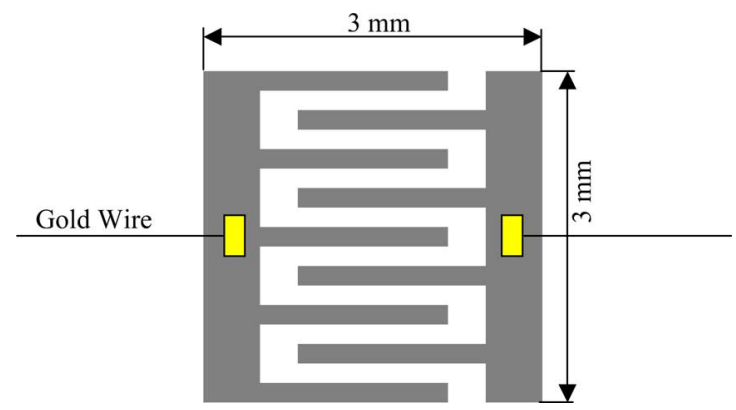

Fig. 4. Schematic diagram of the conductometric transducer pattern.

\section{B. Computerized Gas Calibration System}

The sensor was mounted inside an enclosed environmental cell. Four mass flow controllers (MFCs) were connected to form a single output that supplies gas to the cell. Teflon tubing was used to prevent atmospheric contamination. A constant flow rate of 0.2 liters per minute was delivered via the MFCs. The sensor was exposed to a hydrogen gas pulse sequence with different concentrations of hydrogen gas diluted in synthetic air at different temperatures.

A computerized gas calibration system was used to vary the concentration of $\mathrm{H}_{2}$ gas in synthetic air. The sensor was connected in series with a Keithley 2001 multimeter and a computer was used to log data from the multimeter. The data was related to resistance variation over time. A programmable power supply was connected to the gas calibration system. Elevation

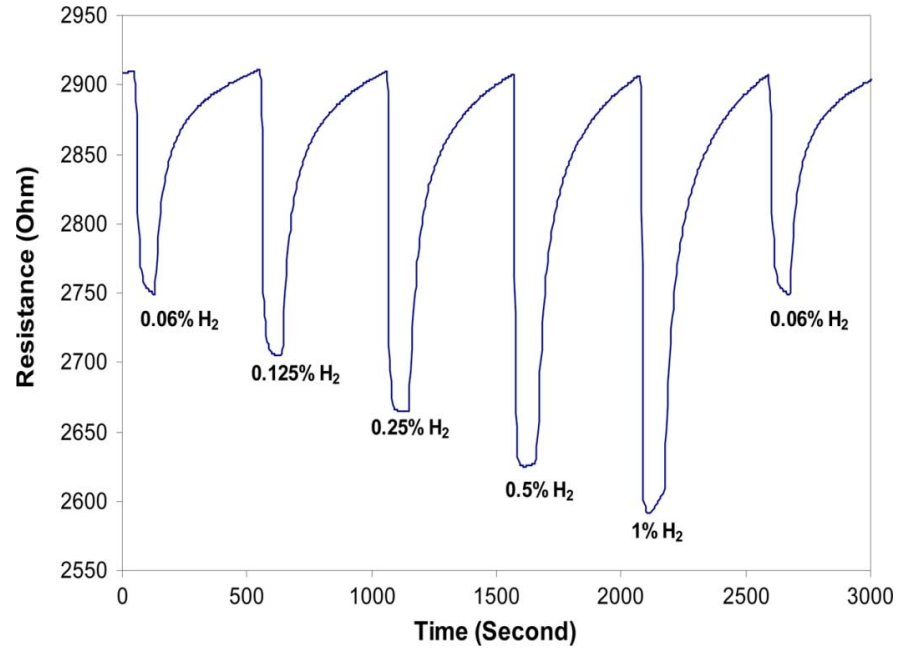

Fig. 5. The dynamic sensor response towards different concentrations of $\mathrm{H}_{2}$.

of the operating temperature was controlled by the same program that supplies gas pulses to the chamber. A $5 \mathrm{~V}$ increment at a time was used to raise the temperature from $22^{\circ} \mathrm{C}-100{ }^{\circ} \mathrm{C}$ gradually, with synthetic air flowing in the chamber. The sensor was allowed to stabilize under synthetic air at each selected operating temperature setting before exposure to the sequence of hydrogen pulses diluted in synthetic air.

\section{RESUltS AND DisUSSION}

The sensor was found to have a short response time of $43 \mathrm{~s}$ upon exposure to a $1 \% \mathrm{H}_{2}$ pulse with a resistance change of $312 \Omega$. Fig. 5 shows the dynamic response of the sensor towards different concentrations of hydrogen gas at room temperature $\left(22^{\circ} \mathrm{C}\right)$. Resistance decreases of $157,205,245$, and $282 \Omega$ were obtained for $0.06 \%, 0.125 \%, 0.25 \%$, and $0.5 \% \mathrm{H}_{2}$ with response times of 75, 72, 61, and $61 \mathrm{~s}$, respectively. Hence, it can be concluded that the highest sensitivity is obtained for $1 \% \mathrm{H}_{2}$ with the shortest response time. Recovery time was fixed at $7 \mathrm{~min}$ under synthetic air after the exposure to each hydrogen pulse.

The polypyrrole nanofiber sensor is reproducible, as shown in Fig. 5, with the start of a new pulse sequence beginning with $0.06 \%$. Testing with different operating temperature settings did not produce any significant change in the sensor response time or its selectivity towards different concentrations of $\mathrm{H}_{2}$ gas. Although the concentration of $\mathrm{H}_{2}$ is doubled in consecutive pulses 


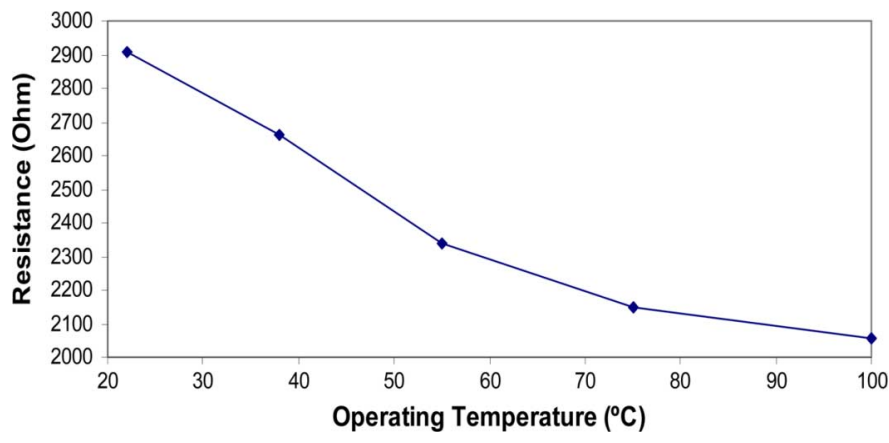

Fig. 6. The effect of elevating the operating temperature on film resistance in synthetic air only (no $\left.\mathrm{H}_{2}\right)$.

the magnitude of the sensor response in Fig. 5 did not double, likely due to saturation of the sensitive layer with hydrogen. This is consistent with similar observation found for hydrogen sensors based on other conducting polymer (such as polyaniline) nanofibers [22].

The dynamic response is generated because of the interaction between the polymer layer and the adsorbed gas molecules. The interaction leads to a heterogeneous charge transfer reaction [23] and hence to a chemical modulation of the polymer doping level, which is directly related to the Fermi level of the organic semiconductor [24]. This effect results in a change in the electronic conductivity or the work function, $\Phi$, of the organic layer. In case of $\Phi$, the polarity of the response depends on the ability of the diffusing gas molecules to exchange charge density with the polymer matrix either by oxidation $(\Delta \Phi>0)$ or reduction $(\Delta \Phi<0)$. The gas molecules behave as electron acceptors and donors, respectively. In Fig. 5, the resistance of PPy nanofibers decreases during the exposure to $\mathrm{H}_{2}$ because hydrogen may act as a reducing gas. Alternatively, a mechanism involving the bridging of conducting polymer chains by hydrogen has been proposed [22].

The redox reaction of PPy is responsible for large changes in its electrical resistance [25]. Most conducting polymers are inherent anion-exchange materials in their conducting form due to the positive charges delocalized over their backbones. It has been shown that PPy is a strong anion exchanger with a capacity of $7.1 \times 10^{-4} \mathrm{~mol} \mathrm{~g}^{-1}$ [26]. Equation (1) describes the redox reaction of PPy, which causes the conductivity changes in PPy
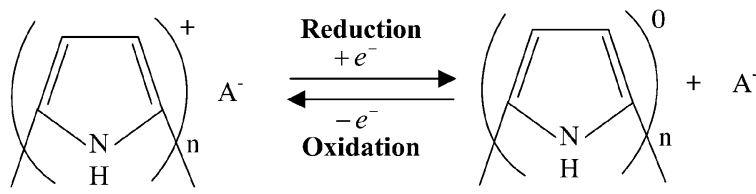

In this work, we also studied the effect of elevating the operating temperature on the dynamic response of PPy nanofibers towards different concentrations of the hydrogen gas/synthetic air mixture. Under synthetic air only, the changes in film resistance as a function of increasing temperature from $22^{\circ} \mathrm{C}-100{ }^{\circ} \mathrm{C}$ are shown in Fig. 6. It can be seen that the film resistance decreases, i.e., the conductivity increases, consistent with an increase in the number of thermally activated carriers. Fig. 7 presents the steady-state sensor response under hydrogen exposure.

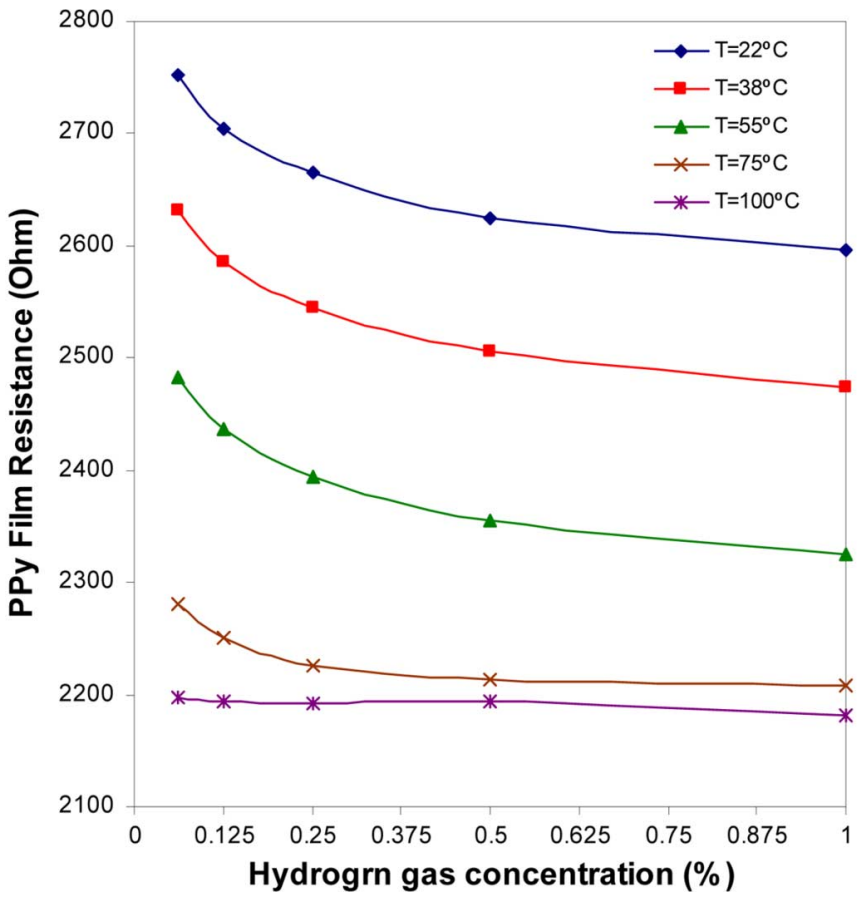

Fig. 7. Variation of the steady-state response of PPy nanofiber sensor as a function of hydrogen gas concentrations.

The nonlinearity of the curves in the temperature range between $22{ }^{\circ} \mathrm{C}-55^{\circ} \mathrm{C}$ can be described by a Langmuir type isotherm as established in the literature [27]. At higher operating temperatures, the response is almost flat, suggesting that the material's response saturates presumably due to the creation of a large number of carriers with high energy moving freely in the polymer chain, thus preventing the polymer from exhibiting its inherent redox reaction behavior. From Fig. 7, it is evident that the conductivity of PPy nanofibers is increasing with temperature elevation and the proposed idea for this observation is that the conductivity increase is due to improvement in the nanostructured film quality because of the temperature increase. At elevated temperatures, the nanostructured film is expected to be smoother comprising dense nanofibrous morphology that has less porosity compared with the film quality at lower temperatures, resulting in an increase of the film conductivity due to the increase of charge carriers hopping between localized states along the polymer chains in accordance with Mott's Variable Range Hopping (VRH) conduction model [28]. The high conductivity of the nanostructured film degrades the sensor response significantly at high temperatures, as shown in Fig. 7, due to the lack of deep penetration of the gas molecules in the bulk of the sensitive layer.

It is worthwhile to mention that humidity has been suppressed in this study through purging the gas chamber with dry synthetic air for $1.5 \mathrm{~h}$ before starting the tests. The synthetic air used constitutes of nitrogen $78 \%$, oxygen $21 \%$, and argon $1 \%$ according to manufacturer data sheet. Diluting hydrogen in synthetic air may produce some $\mathrm{H}_{2} \mathrm{O}$ molecules but high operating temperature maintains $\mathrm{H}_{2} \mathrm{O}$ in the vapor state and purging the cell with dry synthetic air for 7 min after gas exposure eliminates the effect of humidity. Our decision to eliminate the effect of humidity 


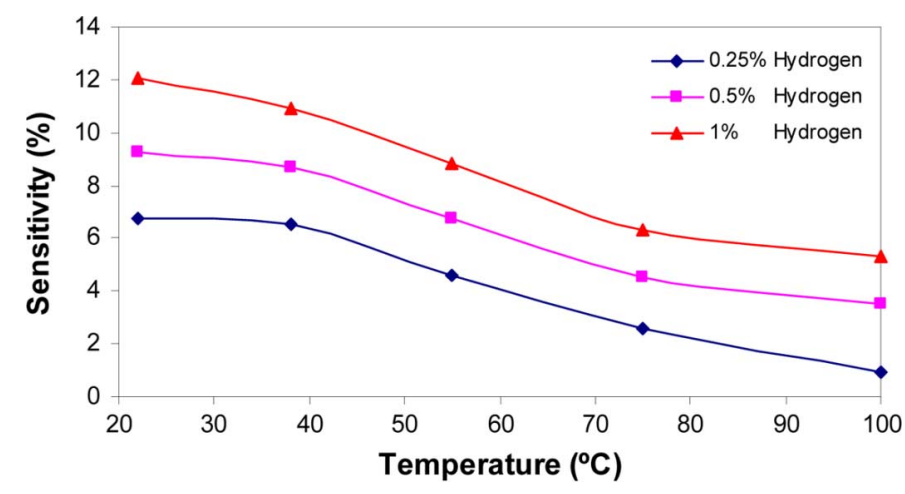

Fig. 8. The conductometric sensor sensitivity as a function of temperature increase for three concentrations of hydrogen gas in synthetic air mixture.

in our study is based on a previous report on the effect of humidity on polyaniline nanofibers that concluded that humidity can impair the sensor response [22].

The conductometric sensor sensitivity $(S)$ is defined by (2)

$$
S=\frac{R_{a}-R_{g}}{R_{g}} \times 100 \%
$$

where $R_{a}$ is the initial resistance of the sensor under synthetic air and $R_{g}$ is the sensor resistance during the exposure to hydrogen gas. In an earlier study, it was shown that variation of the nanofiber film thickness in the range of $0.2-2.0 \mu \mathrm{m}$ did not produce any significant change in sensor sensitivities [11]. This observation was interpreted in terms of the high porosity of the nanofiber films and, hence, high surface to volume ratio which also explains the enhanced sensitivity of these films compared with conventional bulk thin films.

We found that sensitivity of the sensor degrades with increasing temperature. Fig. 8 displays the relation between variable temperature and sensor sensitivity. It can be seen that the best operating temperature for the polypyrrole nanofibers in gas sensing is at room temperature.

\section{CONCLUSION}

Polypyrrole nanofibers have been successfully synthesized using a template-free route. The fibrillar morphology was confirmed using both SEM and TEM. The average diameter of the polypyrrole nanofibers is $\sim 18 \mathrm{~nm}$ with an average length of several micrometers. Elemental analysis revealed a $\mathrm{C} / \mathrm{N}$ ratio of 3.5, which is consistent with the theoretical value for PPy. FT-IR confirms that the structure of the nanofibers is comparable to bulk polypyrrole. A conductometric transducer was fabricated on a sapphire substrate and PPy nanofibers were deposited on its electrode. The sensor performance was analyzed after exposure to different concentrations of hydrogen gas in a synthetic air mixture. A resistance shift of $312 \Omega$ in the presence of a $1 \%$ hydrogen pulse was obtained with a 43 s response time. The sensor sensitivity is largely dependent on the operating temperature, as well as the concentration of the target gas. Optimum sensor performance was found to be at room temperature. However, the sensor maintained its systematic response towards different concentrations of hydrogen gas at elevated temperatures up to $100{ }^{\circ} \mathrm{C}$ despite a slow degradation in sensitivity.

\section{REFERENCES}

[1] C. Nylabder, M. Armgrath, and I. Lundstrom, "An ammonia detector based on a conducting polymer," in Proc. Int. Meeting Chem. Sensors, Fukuoka, Japan, 1983, pp. 203-207.

[2] J. J. Miasik, A. Hooper, and B. C. Tofield, "Conducting polymer gas sensors," J. Chem. Soc., Faraday Trans. 1, vol. 82, pp. 1117-1126, 1986.

[3] G. G. Wallace, G. M. Spinks, and P. R. Teasdale, Conductive Electroactive Polymers. Lancaster, PA: Technomic Pub. Co. Inc., 1997, p. 33.

[4] J. P. Blanc, N. Derouiche, A. El Hadri, J. P. Germain, C. Maleysson, and H. Robert, "Study of the action of gases on a polypyrrole film," Sens. Actuators, B, vol. 1, pp. 130-133, 1990.

[5] P. N. Bartlett and S. K. Ling-Chung, "Conducting polymer gas sensors. Part II: Response of polypyrrole to methanol vapour," Sens. Actuators vol. 19, pp. 141-150, 1989.

[6] P. N. Bartlett and S. K. Ling-Chung, "Conducting polymer gas sensors. Part III: Results for four different polymers and five different vapors," Sens. Actuators, vol. 20, pp. 287-292, 1989.

[7] M. Josowicz and J. Janata, "Suspended gate field-effect transistor modified with polypyrrole as alcohol sensor," Anal. Chem., vol. 58, pp. 514-517, 1986.

[8] M. Josowicz, J. Janata, K. Ashley, and S. Pons, "Electrochemical and ultraviolet spectrochemical investigation of selectivity of potentiometric gas sensors based on polypyrrole," Anal. Chem., vol. 59, pp. 253-258, 1987.

[9] D. M. Liu, J. Aguilar-Hernandez, K. Potje-Kamloth, and H. D. Liess, "A new carbon monoxide sensor using a polypyrrole film grown on an interdigital-capacitor substrate," Sens. Actuators, B, Chem., vol. 41, pp. 203-206, 1997.

[10] S. A. Waghuley, S. M. Yenorkar, S. S. Yawale, and S. P. Yawale, "Application of chemically synthesized conducting-polymer polypyrrole as a carbon dioxide gas sensor," Sens. Actuators, B, Chem., 2007, in press.

[11] J. Huang, S. Virji, B. H. Weiller, and R. B. Kaner, "Nanostructured polyaniline sensors," Chem. Euro. J., vol. 10, pp. 1314-1319, 2004.

[12] V. P. Menon, J. Lei, and C. R. Martin, "Investigation of molecular and supermolecular structure in template-synthesized polypyrrole tubules and fibrils," Chem. Mater., vol. 8, pp. 2382-2390, 1996.

[13] M. Ikegame, K. Tajima, and T. Aida, "Template synthesis of polypyrrole nanofibers insulated within one-dimensional silicate channels: Hexagonal versus lamellar for recombination of polarons into bipolarons," Angew. Chem. Int. Ed., vol. 42, pp. 2154-2157, 2003.

[14] X. Zhang and S. K. Manohar, "Bulk synthesis of polypyrrole nanofibers by a seeding approach," J. Amer. Chem. Soc., vol. 126, pp. 12714-12715, 2004.

[15] G. Li and Z. Zhang, "Synthesis of dendritic polyaniline nanofibers in a surfactant gel," Macromolecules, vol. 37, pp. 2683-2685, 2004.

[16] Y. Yang, J. Liu, and M. Wan, "Self-assembled conducting polypyrrole micro/nanotubes," Nanotechnology, vol. 13, pp. 771-773, 2002.

[17] S. Virji, J. Huang, R. B. Kaner, and B. H. Weiller, "Polyaniline nanofiber gas sensors: Examination of response mechanisms," Nano Lett., vol. 4, pp. 491-496, 2004.

[18] J. Huang, S. Virji, B. H. Weiller, and R. B. Kaner, "Polyaniline nanofibers: Facile synthesis and chemical sensors," J. Amer. Chem. Soc., vol. 125, pp. 314-315, 2003.

[19] G. R. Geier and S. C. Grindrod, "Meso-substituted [34] octaphyrin (1.1.1.0.1.1.1.0) and corrole formation in reactions of a dipyrromethanedicarbinol with 2,2'-bipyrrole," J. Org. Chem., vol. 69, pp. 6404-6412, 2004.

[20] H. D. Tran, K. Shin, W. G. Hong, J. M. D’Arcy, R. W. Kojima, B H. Weiller, and R. B. Kaner, "A template-free route to polypyrrole nanofibers," Macromol. Rapid Commun., vol. 28, pp. 2289-2293, 2007.

[21] T. Hanawa, S. Kuwabata, and H. Yoneyama, "Gas sensitivity of polypyrrole films to $\mathrm{NO}_{2}$," J. Chem. Soc. Faraday Trans. 1, vol. 84, pp. 1587-1592, 1988.

[22] S. Virji, R. B. Kaner, and B. H. Weiller, "Hydrogen sensors based on conductivity changes in polyaniline nanofibers,"J. Phys. Chem. B, vol. 110, pp. 22266-22270, 2006

[23] J. Janata, "Chemical modulation of the electron work function," Anal. Chem., vol. 63, pp. 2546-2550, 1991.

[24] S. M. Sze, Physics of Semiconductor Devices, 2nd ed. New York: Wiley, 1981, p. 22.

[25] T. A. Skotheim, R. L. Elsenbaumer, and J. R. Raynolds, Handbook of Conducting Polymers, 2nd ed. New York: Marcel Dekker, 1998, p. 531 .

[26] H. Ge and G. G. Wallace, "Ion exchange properties of polypyrrole," React. Polym., vol. 18, pp. 133-140, 1992.

[27] P. N. Bartlett and J. W. Gardner, , J. W. Gardner and P. N. Bartlett, Eds., Sensors and Sensory Systems for an Electronic Nose. Norwell, MA: Kluwer, 1991, pp. 31-51.

[28] A. Kaynak, "Electrical conductivity of polypyrrole films at a temperature range of 70 K to 350 K," Mat. Res. Bulletin, vol. 33, pp. 81-88, 1998. 
Laith Al-Mashat (M'07) received the B.Sc. degree in electronics and communications engineering from the University of Technology, Baghdad, Iraq, in 1995. $\mathrm{He}$ is currently working towards the Ph.D. degree at RMIT University, Melbourne, Australia, working on conducting polymer nanostructures for sensing applications.

Since 1995, he worked as an R\&D engineer in several engineering firms. His most recent published work is "Hydrogen gas sensor fabricated from polyanisidine nanofibers deposited on $36^{\circ} \mathrm{YX} \mathrm{LiTaO}_{3}$ layered surface acoustic wave transducer," in BioMEMS and Nanotechnology III, SPIE, vol. 6799, 1, pp. 67991B1-8, 2007.

Mr. Al-Mashat is a Professional Engineer member of the Institution of Engineers, Australia.

Henry D. Tran received the B.S. degree in chemistry from the University of California, Berkeley, in 2003. He is currently working towards the Ph.D. degree in organic chemistry at the University of California, Los Angeles (UCLA).

He joined the UCLA Chemistry Department in 2004 and is currently working under the supervision of Prof. Richard B. Kaner. His research focuses on the development of template-free synthetic routes to nanostructured conducting polymers.

Wojtek Wlodarski (M'72) received the M.Sc.E.E., Ph.D., and D.Sc. degrees from the Warsaw University of Technology, Warsaw, Poland, in 1962, 1971, and 1980 , respectively.

He has worked in the areas of sensor technology and instrumentation for over 40 years. He is a Professor with RMIT University, Melbourne, Australia, and heads the Sensor Technology Laboratory at the School of Electrical and Computer Engineering. He has published four books and monographs and over 400 papers and holds 29 patents.
Richard B. Kaner received the Ph.D. degree in inorganic chemistry from the University of Pennsylvania, Philadelphia, in 1984, followed by two and a half years of Postdoctoral Research at the University of California, Berkeley.

$\mathrm{He}$ is a Professor of Chemistry and a Professor of Materials Science and Engineering at the University of California, Los Angeles (UCLA). He joined UCLA in 1987 as an Assistant Professor, earned tenure in 1991, and became a Full Professor in 1993. He has published over 175 papers in top peer reviewed journals and holds ten U.S. patents with ten more pending.

Dr. Kaner has received awards from the National Science Foundation (Presidential Young Investigator Award), the American Chemical Society (BuckWhitney Research Award and Exxon Solid State Chemistry Fellowship), as well as awards from the Dreyfus, Fulbright, Guggenheim, Packard and Sloan Foundations for his work on new routes to materials including intercalation compounds, carbon nanoscrolls, graphene, fulleride superconductors, and conducting polymers nanowires.

Kourosh Kalantar-Zadeh (M'94) received the M.Sci. degree in telecommunications from Tehran University, Tehran, Iran, in 1997 and the Ph.D. degree in micro/nanoelectronics and bioelectronics from RMIT University, Melbourne, Australia, in 2002.

$\mathrm{He}$ is currently a Senior Lecturer at the School of Electrical and Computer Engineering, RMIT University. He has also published a book titled Nanotechnology Enabled Sensors (New York: Springer, 2008). He holds three patents and has published over 120 scientific papers in refereed journals and in the proceedings of international conferences. His research interests include chemical and biochemical sensors, nanotechnology, microelectromechanical systems, thermoelectric materials, electronic circuits, and microfluidics. 